Conclusion Contrary to what is found in similar studies in SSA, where hypertension is highest in urban areas, we found that rural residence, abdominal obesity among men and generalised obesity among women were the most important predictors of hypertension. Intervention to reduce hypertension could be further targeted towards rural areas. Sensitisation campaigns should promote awareness of the risk factors, especially on the importance of maintaining a healthy weight.

\section{OP98 REVISITING EARLY LIFE DEPRIVATION AND CARDIOVASCULAR DISEASE: AN ECOLOGICAL STUDY OF HISTORICAL TRENDS IN ECONOMIC DEVELOPMENT AND CURRENT CARDIOVASCULAR MORTALITY IN 1577 BRAZILIAN MUNICIPALITIES}

PAC Mallinson*, S Kinra. Department of Non-communicable Disease Epidemiology, London School of Hygiene and Tropical Medicine, London, UK

\subsection{6/jech-2017-SSMAbstracts.97}

Background Ecological findings from Europe in the 1970s demonstrated an interaction between deprivation during early life and adult cardiovascular disease (CVD) mortality. These findings hold renewed significance today in the context of emerging epidemics of CVD in rapidly developing countries. If generalizable to such settings, understanding of the interaction between early life deprivation and CVD mortality might improve disease projections and targeting of resources to high risk areas. To investigate this, we studied economic development rates since 1940 and current CVD mortality in Brazil.

Hypothesis Currently, higher GDP/capita is associated with higher CVD mortality at municipality level in Brazil. We hypothesised that if deprivation was a risk factor for CVD mortality during the early life period, municipalities which have undergone the greatest shifts from low to high GDP/capita in the past 50-70 years would have higher CVD mortality rates today than those with consistently high or low GDP/ capita.

Methods We used municipality-level data on deaths, demographics and gross domestic product (GDP) from the Brazilian Institute of Geography and Statistics. Our primary outcome was CVD mortality rate in 2005-14, defined and adjusted according to the WHO Global Burden of Disease protocol. We compared the trajectory of municipality GDP/capita between time of birth and time of death, defined by tertiles of GDP/capita at each time point. Analyses were age-standardised and stratified by sex. Municipalities were grouped to reflect 1940 s borders and excluded where this was not possible. We conducted analyses on $\mathrm{R}$.

Results In 1557 included municipalities, 367 had a low-low GDP/capita trajectory, 44 had low-high and 329 had highhigh. Age-adjusted CVD mortality rates for $>50$ year-olds, per 100000 person years, in low-low, low-high and high-high trajectory municipalities, respectively, were 656.7 (95\% CI: 636.2, 677.2), 758.2 (95\%CI: 713.8, 802.5) and $821.9(95 \%$ CI: $810.7,833.2)$ among men (p-value trend test <0.001); and 447.0 (95\%CI: 430.5, 463.4), 414.4 (95\%CI: 383.5, $445.3)$ and 449.5 (95\%CI: 442.0, 457.0) among women (pvalue trend test $>0.1$ ). These findings were not substantially altered in sensitivity analyses checking for the potential effects of internal migration.

Conclusion Contrary to what we hypothesised, Brazilian municipalities which have shifted from low to high GDP/ capita did not exhibit higher rates of CVD mortality than consistently high or low municipalities. This reminds us to be cautious extrapolating evidence generated in high-income settings to rapidly developing settings where social and economic contexts surrounding CVD differ markedly. Further individuallevel studies with robust designs are needed, as inference from ecological studies has limitations.

\section{OP99 PSYCHOLOGICAL DISTRESS AND INCIDENT STROKE RISK IN THE 45 AND UP STUDY}

${ }^{1,2} \mathrm{CA}$ Jackson*, ${ }^{3} \mathrm{CLM}$ Sudlow, ${ }^{2} \mathrm{GD}$ Mishra. ${ }^{1}$ Usher Institute of Population Health Sciences and Informatics, University of Edinburgh, Edinburgh, UK; ${ }^{2}$ School of Public Health, University of Queensland, Brisbane, Australia; ${ }^{3}$ Centre for Clinical Brain Sciences, University of Edinburgh, Edinburgh, UK

\subsection{6/jech-2017-SSMAbstracts.98}

Background Few studies have reported on the association between psychological distress and stroke risk, with most investigating the effect of psychological distress on stroke mortality rather than incidence. We investigated whether psychological distress is associated with stroke incidence in a large population-based cohort study and examined whether associations differed by gender, age-group and pathological stroke type.

Methods We included 228955 participants without prior stroke from the New South Wales 45 and Up Study. Baseline psychological distress was measured using the 10-item Kessler psychological distress Scale and categorised as low, medium, and high/very high. We identified incident stroke through linkage to hospital admission and mortality records. We analysed men and women separately, using cox survival analysis to obtain unadjusted and adjusted hazard ratios (HRs) with 95\% confidence intervals (CIs) for the association between psychological distress and all stroke and pathological stroke types. We serially adjusted for groups of confounders, including: sociodemographic factors; lifestyle factors; clinical disease history; family history of cardio- and cerebrovascular disease; physical disease co-morbidity (Charlson comorbidity index); and (among women) menopausal status and current hormone replacement therapy and oral contraceptive use.

Results During 1,075,057 person-years of follow-up (mean follow-up time $4.7 \pm 0.98$ years), we identified 2682 incident strokes among men and women. In men, medium and high/ very high psychological distress was associated with a $10 \%$ and $34 \%$ increased risk of stroke compared to low psychological distress (fully adjusted HRs 1.10, 95\% CI 0.95, 1.26 and 1.34, 95\% CI 1.11, 1.62, respectively). Similar effect estimates were observed in women (fully adjusted HRs for medium and high/very high versus low psychological distress: $1.17,95 \% \mathrm{CI}$ $1.00,1.37$ and $1.44,95 \%$ CI 1.18, 1.77). Effect estimates were similar across age-groups and pathological stroke type, in both men and women.

Conclusion Psychological distress is strongly associated with increased stroke risk, even after adjusting for a wide range of confounding factors. Further investigation is needed to establish whether this relationship is causal and to determine the underlying mechanism(s). Meanwhile, study findings support the need for renewed efforts: to encourage people with mental ill-health to seek medical help; for better screening and treatment for mental health conditions (which might itself reduce cerebrovascular and cardiovascular disease risk); and to 
encourage screening for established cerebrovascular risk factors in this high-risk, vulnerable group.

\section{OP100 THE COST-EFFECTIVENESS AND EQUITY OF THE NHS HEALTH CHECKS CARDIOVASCULAR DISEASE PREVENTION PROGRAMME: A MICROSIMULATION USING REAL-WORLD DATA FROM A DEPRIVED NORTHERN CITY}

${ }^{1} \mathrm{~B}$ Collins*, ${ }^{1} \mathrm{C}$ Kypridemos, ${ }^{2} \mathrm{P}$ Parvulescu, ${ }^{2} \mathrm{R}$ Gosling, ${ }^{1} \mathrm{~S}$ Capewell, ${ }^{1} \mathrm{M}$ O'Flaherty. ${ }^{1}$ Department of Public Health and Policy, University of Liverpool, Liverpool, UK; ${ }^{2}$ Public Health Department, Liverpool City Council, Liverpool, UK

\subsection{6/jech-2017-SSMAbstracts.99}

Background The NHS Health Check Programmes' stated objective is the early identification of otherwise healthy people at high risk of cardiovascular disease (CVD) and diabetes. However, the programme's effectiveness, cost-effectiveness, and equity are still contested. This study therefore aimed to determine whether Health Checks (HCs) are cost-effective and equitable in a city with high levels of deprivation and CVD.

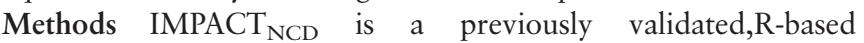
dynamic stochastic microsimulation policy model. We calibrated this model to Liverpool demographics, risk factor exposures, and CVD epidemiology. We modelled the current implementation of HCs using local and national data on effectiveness, costs, and participant risk profiles. Disease costs and health state utilities were drawn from standard sources and discounted at $3.5 \%$ annually using a healthcare perspective. We quantified the uncertainty of model outputs using secondorder Monte Carlo simulation, and report 95\% uncertainty intervals.

We modelled three fifteen year scenarios from 2017 to 2031:

A) continuing the current implementation of HCs;

B) an optimal implementation of HCs assuming optimal coverage, uptake, treatment and lifestyle change;

C) combining scenario A with structural policies targeting dietary consumption of salt, sugar, fruit and vegetables.

We compared all three scenarios with a counterfactual of no HCs, and conducted a rigorous sensitivity analysis.

Results The model suggested that over 15 years the CVD cases prevented or postponed would be approximately 310 (40-734) for scenario A, 870 (327-1,397) for scenario B, and $1740(815-2,939)$ for scenario C.

Cumulative discounted net costs and quality-adjusted life years (QALYs) gained for the three scenarios respectively would be $+£ 2.1 \mathrm{~m}(£ 1.5 \mathrm{~m}-+£ 4.8 \mathrm{~m})$ and +90 QALYs $(-124-+376)$ for $\mathrm{A} ; \quad+£ 1.4 \mathrm{~m} \quad(£ 6.1 \mathrm{~m}-+£ 6.6 \mathrm{~m})$ and +434 QALYs $(-76-+1,133)$ for $\mathrm{B}$; or $£ 16.9 \mathrm{~m}$ $(£ 33.2 \mathrm{~m}-£ 5.9 \mathrm{~m})$ and $+2,871$ QALYs $(+1,355-+4,830)$ for $\mathrm{C}$.

We estimated the probability of scenarios $\mathrm{A}$ and $\mathrm{B}$ being cost-effective by 2031 at $25 \%$ and $74 \%$ respectively, valuing each QALY at $£ 20000$.

Scenario C would become cost saving by 2030 .

Scenario A may increase existing health inequalities; $\mathrm{B}$ is likely to be neutral, while $\mathrm{C}$ would substantially decrease inequalities.

In extensive sensitivity analyses, the direction of the results did not change when the discount rate was varied from $0 \%-$ $6 \%$, nor when the time horizon was increased to 20 years.
Conclusion Current NHS Health Checks implementation appears neither equitable nor cost-effective. The addition of structural policies proved equitable and cost saving. Future research might now seek to identify the optimal combination of structural policies at local level.

\section{SSM annual scientific meeting 2017}

\section{Plenary $(\mathrm{PL})$ presentations}

\section{PL01 THE IMPACT OF CO-LOCATED WELFARE ADVICE IN PRIMARY HEALTHCARE SETTINGS ON MENTAL HEALTH AND HEALTH SERVICE USE: A MIXED METHODS EVALUATION}

${ }^{1} \mathrm{C}$ Woodhead*, ${ }^{2} \mathrm{M}$ Khondoker, ${ }^{1} \mathrm{R}$ Raine. ${ }^{1}$ Applied Health Research, University College London, London, UK; ${ }^{2}$ Medicine and Health Sciences, University of East Anglia, Norwich, UK

\subsection{6/jech-2017-SSMAbstracts. 100}

Background Co-locating welfare advice services in primary healthcare settings has been one approach to tackling health inequalities by increasing income among socially deprived individuals. It is also hoped to relieve pressure on general practices in supporting patients with 'non-clinical' needs. Previous evaluations have been methodologically limited and lack theoretical underpinning. We aimed to examine the impact of colocated welfare benefits and debt advice on mental health and primary care service use, and to develop theory linked to pathways of effect.

Methods A prospective, controlled quasi-experimental study with an embedded qualitative component was carried out (December 2015-December 2016) in eight intervention and nine comparator sites across North Thames, London. Beforeand-after quantitative data were collected via self-report questionnaires. Comparison group members were propensity score weighted for analyses. Outcomes included change in symptoms of common mental disorder (CMD) (12-item General Health Questionnaire), well-being (Shortened Warwick and Edinburgh Mental Well-being Scale), three-month GP consultation rate and financial strain. Data from qualitative interviews with 24 primary care staff, funders and advice providers were analysed using a modified realist evaluation approach to understand how co-located welfare advice could influence practice outcomes.

Results For the quantitative study, $n=285$ and $n=633$ individuals were recruited into advice and comparison groups respectively at baseline. $72 \%$ and $84 \%$ were retained at 3 month follow-up. Relative to controls, CMD caseness reduced significantly among female and Black/Black British advice recipients. Individuals whose advice resulted in positive outcomes demonstrated significantly improved well-being scores. Significant reduction in financial strain overall but no change in threemonth consultation rate was found. Per capita, advice recipients received $£ 15$ per $£ 1$ of funder investment. Qualitative findings were used to inform underlying theory linking service activity to general practice outcomes. These were reduced GP consultations for 'non-clinical' issues and reduced practice staff time supporting patients with such issues. The findings revealed key implementation, context and agency factors that facilitated or hindered the potential for co-located advice to influence these outcomes. 